\title{
Estudos Secundários: Compartilhando Experiências Práticas de Revisões da Literatura em IHC
}

\author{
Luis Rivero \\ PPGCC - UFMA \\ São Luis / MA / Brasil \\ luisrivero@nca.ufma.br
}

\author{
Rodrigo Santos \\ PPGI - UNIRIO \\ Rio de Janeiro / RJ / Brasil \\ rps@uniriotec.br
}

\author{
Davi Viana \\ PPGCC - UFMA \\ São Luis / MA / Brasil \\ davi.viana@1sdi.ufma.br
}

\begin{abstract}
RESUMO
A revisão da literatura é uma importante etapa na execução de uma pesquisa. Cada vez mais tais revisões estão sendo preparadas através de métodos sistemáticos, por meio de estudos secundários. A realização de estudos secundários permite: identificar publicações relevantes; identificar novas oportunidades de investigação; sumarizar as evidências existentes; e possibilidade de replicação futura, incluindo novas publicações. Este minicurso apresenta o processo para a realização de estudos secundários em IHC para ser aplicado por alunos, pesquisadores e/ou profissionais interessados em identificar o estado da arte de algum tópico de pesquisa na área. Na parte teórica, são apresentados os objetivos e fundamentos de estudos secundários, incluindo os conceitos e etapas necessários para sua execução. Na parte prática, ferramentas de apoio e exemplos de aplicação são explorados em atividades com os participantes.
\end{abstract}

\section{PALAVRAS-CHAVE}

Revisão Sistemática da Literatura, Mapeamento Sistemático da Literatura, Estudos Secundários, Minicurso

\section{APRESENTAÇÃO}

Segundo Mafra et al. [1], em uma metodologia de pesquisa, a revisão da literatura permite analisar as publicações prévias em um tópico de pesquisa, identificando o conhecimento disponível sobre a área investigada. Enquanto estudos primários apresentam resultados experimentais para responder uma questão de pesquisa, estudos secundários operacionalizam revisões da literatura e visam agregar e sintetizar os resultados de estudos primários [2].

Kuhrmann et al. [3] afirmam que os estudos secundários podem ser classificados em: (a) Revisões Sistemáticas da Literatura (RSLs), que têm por objetivo identificar, avaliar e interpretar todos os estudos experimentais relevantes a um determinado tópico de pesquisa, de maneira comparativa; e (b)

Permission to reproduce or distribute, in whole or in part, material extracted from this work, verbatim, adapted or remixed, as well as the creation or production from the content of such work, is granted without fee for non-commercial use, provided that the original work is properly credited.

IHC 2019 - TRILHA MINICURSOS, Outubro 21-25, 2019, Vitória, Brasil. In Anais Estendidos do XVIII Simpósio Brasileiro sobre Fatores Humanos em Sistemas Computacionais. Porto Alegre: SBC.

(C) 2019 by the author(s), in accordance with the terms of the Creative Commons Attribution-NonCommercial 4.0 International Public License (CC BY-NC 4.0).
Mapeamentos Sistemáticos da Literatura (MSLs), que permitem classificar os tópicos estudados dentro de uma área de pesquisa. A palavra "sistemático" indica que procedimentos metodológicos são aplicados para diminuir as ameaças à validade dos resultados decorrentes da realização destas revisões [4].

Devido aos benefícios decorrentes da realização de estudos secundários, os pesquisadores em IHC no Brasil têm aderido à utilização de RSLs e MSLs para analisar o estado da pesquisa na área. No entanto, pesquisadores novatos ou com pouca experiência na realização de estudos secundários se deparam com dificuldades cujas soluções específicas não são completamente descritas nos guias para execução de estudos secundários [3].

Este minicurso tem por objetivo prover conhecimentos, discussões e reflexões, de forma didática, sobre a teoria e prática necessárias para a execução de estudos secundários. O material do minicurso serve de referência para alunos de graduação e pósgraduação em IHC, cobrindo um tópico relevante (condução de estudos secundários), facilitando a disseminação do conhecimento e a consolidação das pesquisas em IHC. Espera-se apresentar uma visão geral e formativa do processo de execução de estudos secundários, fornecendo exemplos de aplicação de RSLs e MSLs para responder questões de pesquisas na área.

\section{SUMÁRIO ESTENDIDO}

Este minicurso do IHC 2019 tem duração de três horas: (a) uma hora de exposição de conceitos; e (b) duas horas de atividade supervisionada, em que são realizadas atividades práticas para fixação dos conteúdos. Os tópicos do minicurso são descritos abaixo, seguindo temas propostos por Kitchenham et al. [5]:

Tópico 1 - Introdução: Conceitos sobre metodologia de pesquisa, estudos primários, estudos secundários e tipos de estudos em ambas categorias. Apresentação de justificativa e motivação para a realização de estudos secundários em IHC.

Tópico 2 - Processo de Execução de Estudos Secundários: Planejamento de estudos secundários, incluindo: identificação da necessidade do estudo, definição do tipo de estudo secundário, definição da questão de pesquisa, definição da estratégia de busca, definição de critérios para incluir ou excluir uma publicação, avaliação da qualidade da publicação considerada, estratégia para extração de dados, processo de síntese dos dados coletados, definição de estratégia de reporte de dados, e definição do protocolo. Execução e publicação dos resultados. 
Tópico 3 - Ferramentas: Ferramentas de apoio ao processo de execução de estudos secundários, incluindo definição de protocolo e execução de revisão utilizando as ferramentas existentes: StArt (http://lapes.dc.ufscar.br/tools/start_tool) e PARSIFAL (https://parsif.al).

Tópico 4 - Exemplo de Revisões Sistemáticas em Interação Humano-Computador: Apresentação de casos onde foram aplicadas revisões e/ou mapeamentos sistemáticos da literatura em IHC. Os exemplos são dos autores e de publicações científicas.

\section{PÚBLICO-ALVO}

Os participantes deste minicurso devem ser estudantes, profissionais e/ou professores que tenham interesse em conhecer e aplicar o processo de planejamento e execução e análise de resultados de estudos secundários na área de IHC para embasar pesquisas científicas e/ou desenvolvimento de novas tecnologias.

Este tema deve ser interessante para este público, visto que a execução de estudos secundários traz inúmeros benefícios para as pesquisas científicas e benefícios na proposição de novas tecnologias. Além disso, a execução adequada de um estudo secundário também possibilita a publicação de um artigo em veículos científicos. Nesse sentido, este minicurso busca apresentar os conceitos deste tema levando em consideração aspectos práticos, contribuindo na melhoria dos trabalhos submetidos e publicados pela comunidade de IHC.

Há diversos desafios na execução de estudos secundários, que precisam ser tratados para beneficiar as áreas da Computação, incluindo IHC. Alguns desafios são: (1) definição adequada das bibliotecas digitais; (2) definição da string de busca de modo que ela cubra o maior número de publicações de interesse; (3) seleção e extração adequada dos estudos primários; (4) síntese dos resultados do estudo secundário; e (5) definição adequada do tipo de revisão. Considerando esses desafios, este minicurso almeja uma reflexão e maior compreensão sobre a condução dos estudos secundários, objetivando sempre melhorar a qualidade dos trabalhos publicados pela comunidade de IHC.

\section{BIOGRAFIA DOS AUTORES}

Luis Rivero - É doutor em Informática pela UFAM e Professor Adjunto do Departamento de Informática da UFMA, onde atua em cursos de graduação e como colaborador do Programa de PósGraduação em Ciência da Computação. Possui experiência na execução de mapeamentos sistemáticos da literatura em IHC. Possui experiência na realização de estudos experimentais, com ênfase na realização de análises quantitativas e qualitativas, necessárias na execução de mapeamentos sistemáticos.

CV Lattes: http://lattes.cnpq.br/8534667641706692

Rodrigo Santos - Professor Adjunto do Departamento de Informática Aplicada e membro efetivo do Programa de PósGraduação em Informática da UNIRIO. Doutor em Engenharia de Sistemas e Computação pela COPPE/UFRJ. Foi pesquisador visitante na University College London. Possui experiência na execução de mapeamentos sistemáticos em Engenharia de
Software, Sistemas de Informação e IHC. Possui experiência na realização de estudos experimentais, com ênfase na realização de análises quantitativas e qualitativas. Por fim, possui experiência em ministrar palestras, minicursos e tutoriais em diversos eventos da SBC, incluindo publicação de capítulos.

CV Lattes: http://lattes.cnpq.br/8613736894676086

Davi Viana - É Doutor em Informática pela UFAM e Professor Adjunto da UFMA, onde atua em cursos de graduação (Bacharelado Interdisciplinar em Ciência e Tecnologia e Bacharelado em Engenharia da Computação) e no Programa de Pós-Graduação em Ciência da Computação. Possui experiência na execução e análise de mapeamentos sistemáticos da literatura na área de Engenharia de Software. Possui experiência em Engenharia de Software Experimental com ênfase na realização de estudos qualitativos. Adicionalmente, conhece técnicas de síntese de dados de mapeamentos sistemáticos.

CV Lattes: http://lattes.cnpq.br/9297257833779277

\section{INFORMAÇÕES ADICIONAIS}

Duração do minicurso - 3 Horas.

Idioma em que será ministrado - Português.

Infraestrutura e materiais necessários e desejáveis - (a) Computador - para a apresentação de slides, realização de exercícios e apresentação prática de ferramentas de apoio ao planejamento e execução de estudos secundários; (b) Projetor multimídia - para a apresentação dos materiais preparados para o minicurso; (c) Computadores com acesso à Internet - para que os participantes possam realizar as atividades de fixação da parte conceitual do minicurso; e (d) Documentos impressos para preenchimento e discussão (relacionados a: protocolo, formulário de extração, entre outros), permitindo uma abordagem desplugada no momento da realização de algumas atividades do minicurso.

\section{REFERÊNCIAS}

[1] S. Mafra, R. Barcelos e G. Travassos. 2006. Aplicando uma Metodologia Baseada em Evidência na Definição de Novas Tecnologias de Software. In Proceedings of the 20th Brazilian Symposium on Software Engineering (SBES).

[2] F. Shull, J. Carver e G. Travassos. 2001. An empirical methodology for introducing software processes. ACM SIGSOFT Software Engineering Notes, 26, 5 (2001), 288-296.

[3] M. Kuhrmann, D. M. Fernández e M. Daneva. 2017. On the pragmatic design of literature studies in software engineering: an experience-based guideline. Empirical Software Engineering 22, 6 (2017), 2852-2891.

[4] C. Wohlin, P. Runeson, M. Höst, M. C. Ohlsson, B. Regnell e A. Wesslén. 2012. Experimentation in software engineering. Springer Science \& Business Media.

[5] B. A. Kitchenham, D. Budgen e P. Brereton. 2016. Evidence-based software engineering and systematic reviews. CRC Press: New York. 426p. 